Meta

Journal des traducteurs

Translators' Journal

\title{
Aspects pédagogiques de la traduction littéraire
}

\section{Denis G. Gauvin et Daniel Slote}

Volume 33, numéro 2, juin 1988

L'enseignement de la traduction au Canada - Teaching Translation

in Canada

URI : https://id.erudit.org/iderudit/002830ar

DOI : https://doi.org/10.7202/002830ar

Aller au sommaire du numéro

Éditeur(s)

Les Presses de l'Université de Montréal

ISSN

0026-0452 (imprimé)

1492-1421 (numérique)

Découvrir la revue

Citer cet article

Gauvin, D. G. \& Slote, D. (1988). Aspects pédagogiques de la traduction littéraire. Meta, 33(2), 183-199. https://doi.org/10.7202/002830ar d'utilisation que vous pouvez consulter en ligne.

https://apropos.erudit.org/fr/usagers/politique-dutilisation/ 


\section{ASPECTS PÉDAGOGIQUES DE LA TRADUCTION LITTÉRAIRE}

DENIS G. GaUVIN ET DANIEL Slote Université de Montréal, Montréal, Québec

\section{"MAIS JE L'AI TROUVÉ DANS LE DICTIONNAIRE "}

Les recréations contextuelles prouvent bien que la connaissance de toutes les équivalences lexicales ou syntagmatiques consignées dans les dictionnaires bilingues ne suffirait pas pour traduire.

Jean Delisle (1981) : " De la théorie à la pédagogie : réflexions méthodologiques ", l'Enseignement de l'interprétation et de la traduction, E.U.O.

Combien d'étudiants, dans le cadre d'un cours de traduction littéraire ${ }^{1}$, ont justifié ainsi une des " trouvailles " qui émaillaient leur travail et que le pédagogue avait, sans vergogne, contestée? Le malheur est que, en traduction littéraire, on appréhende trop souvent le texte à traduire dans une perspective purement lexicale, sans s'attacher à effectuer une analyse du discours en tant que telle. Mais est-ce là la panacée qui permet de réfuter la thèse selon laquelle la traduction est impossible ? En effet, comme le fait remarquer Mounin (1983:8)

L'activité traduisante pose un problème théorique à la linguistique contemporaine : si l'on accepte les thèses courantes sur la structure des lexiques, des morphologies et des syntaxes, on aboutit à professer que la traduction devrait être impossible. (...)

D'autres auteurs sont plus catégoriques : ainsi, d'après Wilhelm von Humboldt (Fedorov 1953) :

Toute traduction me paraît être incontestablement une tentative de résoudre une tâche irréalisable.

Friedrich von Schlegel (Fedorov 1953) se fait l'écho de ces paroles :

La traduction est un duel à mort où périt inévitablement celui qui traduit ou celui qui est traduit.

L'objection la plus forte contre la possibilité même de toute traduction provient de la critique à laquelle un certain nombre de linguistes modernes, tels que Saussure, Harris, Bloomfield, Hjelmslev, ont soumis la notion classique du sens d'un énoncé. Saussure (1982 : 97, 161) rejette d'emblée la notion de langue répertoire.

Pour certaines personnes, la langue ramenée à son principe essentiel est une nomenclature, c'est-à-dire une liste de termes correspondant à autant de choses (...). Cette conception suppose des idées toutes faites préexistant aux mots. (...) Mais si ces mots étaient chargés de représenter des concepts donnés d'avance, ils auraient chacun, d'une langue à l'autre, des correspondants exacts pour le sens : or il n'en est pas ainsi. 
Selon Bloomfield (1933 : 74) :

Notre connaissance du monde dans lequel nous vivons est si imparfaite que nous ne pouvons que rarement rendre un compte exact de la signification d'un énoncé.

La théorie bloomfieldienne en matière de sens nie donc soit la légitimité théorique, soit la possibilité pratique de toute traduction. En effet, le sens étant inaccessible, il est impossible de déterminer si le traducteur l'a fait passer ou non dans la langue cible.

Toutefois, il convient de souligner que ces personnes étaient des linguistes, préoccupés au premier chef par les lois de fonctionnement du langage, par les faits de langue, en dehors de toute préoccupation normative. Von Humboldt et von Schlegel étaient des monadistes, c'est-à-dire des tenants de la théorie suivant laquelle les langues sont des monades qui établissent des relevés contradictoires de la réalité. ("Nous disséquons la nature selon les directives imposées par notre langue maternelle " [Steiner $1978: 93$ ].) Si une telle hypothèse se vérifiait, comment la communication pourrait-elle passer d'une langue à une autre ? Comment apprendre une seconde langue, comment entrer dans un autre univers linguistique au moyen de la traduction? On ne peut certes pas nier que de tels transferts soient courants.

C'est justement à la communication interlinguale et aux mécanismes intellectuels qui la sous-tendent que s'intéresse le traductologue. Ainsi, pour le pédagogue, il est primordial d'isoler ces mécanismes, de les disséquer, de façon à les inculquer aux candidats traducteurs, plutôt que de se borner à présenter ses solutions de traduction d'un texte littéraire, si géniales soient-elles. Par la même occasion, il doit " déblayer le terrain "en ce qui concerne les différentes théories relatives à la traduction littéraire qui, comme nous l'avons souligné plus haut, sont de facture plus linguistique et, de ce fait, plus diffciles à appliquer dans la pratique.

Toutefois, avant d'aller plus loin, il est essentiel de s'interroger sur la façon dont on devient traducteur littéraire. Pour schématiser, nous avons cerné un certain nombre de catégories de traducteurs types.

L'expérience prouve que, malheureusement, encore trop de gens s'improvisent traducteurs littéraires. (Nous entendons par là les gens qui ne possèdent même pas un bagage littéraire suffisant.) Certaines des premières traductions de textes de Zola ou de Dickens sombrent le plus souvent dans le ridicule. Plus près de nous, il suffit de penser à la traduction aberrante de la Peste, de Camus, par Gilbert Stuart, dont les coupures, les faux sens et les inepties de tous ordres font les délices des candidats traducteurs dans maintes écoles de traduction. Quand "l'instinct " de M. Stuart lui fait défaut devant une difficulté apparemment infranchissable, eh bien! il saute tout simplement le passage "rétif ". Il procède sans vergogne de la même façon devant des obstacles mineurs mais inopportuns : en les supprimant, en les rayant du texte à traduire. Signalons au passage que ces exemples d'excès traductionnels ne sont pas l'apanage de traductions ayant l'anglais ou le français comme langue de départ ou d'arrivée. Il suffit, pour s'en convaincre, de jeter un coup d'œil - elles ne méritent pas plus - sur les traductions d'auteurs russes, tchèques, etc. Qu'une " secrétaire traductrice " sans bagage " traduise " une lettre commerciale pour son patron à l'entière satisfaction de ce dernier, tant mieux ! Mais le texte littéraire et ses multiples complexités reste et doit rester hors de sa portée. Ce sont ces personnes qui ont le choc de leur vie lorsqu'elles s'inscrivent à un cours du soir en traduction pour " parfaire » leurs connaissances. Combien de fois le pédagogue a-t-il entendu poser, d'un air pincé, voire outré, la question suivante : "Mais Monsieur, comment se fait-il que vous n'ayez pas aimé ma traduction de tel passage ? Pourtant, j'ai tous les dictionnaires imaginables à la maison! » 
Cette catégorie n'étant manifestement pas pertinente aux fins du présent article, nous la passerons sous silence. En effet, ce qui nous préoccupe ici au premier chef, c'est la « formation" du traducteur, sa "préparation". Mais en quoi consiste cette "préparation $» ?$

La première catégorie - pertinente - de gens "préparés » est constituée de ce que nous appellerons les « littéraires actifs ", c'est-à-dire des producteurs d'œuvres littéraires à part entière qui se sont adonnés à la traduction à un moment de leur carrière d'écrivain. Deux exemples frappants sont sans conteste Baudelaire et Yourcenar. Ces deux auteurs se situent d'ailleurs aux antipodes de la traduction littéraire dans leur façon de procéder : le premier fait un genre de "recréation littéraire ", - " absorption followed by recreation ", pour reprendre la terminologie de Félix Boillot (Horguelin 1981), - bref, il recrée in toto l'œuvre de l'auteur dans l'esprit d'une langue différente, " de façon que les lecteurs étrangers éprouvent, autant que faire se peut, les mêmes émotions que les indigènes à la lecture de l'original " (Horguelin 1981). Le traducteur se pénètre intuitivement du texte, le ressent avec une vive acuité d'émotion et le ressuscite dans un autre idiome. Idéalement, le texte d'arrivée est chargé d'une puissance d'évocation équivalente. Ainsi, comme le souligne Boillot (Horguelin 1981) :

La fidélité au détail n'a plus la même importance car le traducteur cherche à faire aussi bien que son modèle plutôt que de viser à faire exactement la même chose. Comme il s'agit d'une véritable transfusion de sang, une certaine consanguinité entre l'auteur et le traducteur est indispensable. En ce domaine, pour réussir, il faut plus que du génie - un génie supérieur presque à celui de l'original lui-même, car le traducteur entre dans la lice tout chargé de chaînes.

Baudelaire, en tant que traducteur, a souvent été considéré comme un parangon, le parfait exemple du traducteur qui s'est "mis dans la peau de l'auteur ». Selon d'aucuns, c'est grâce à lui que l'œuvre de Poe a connu une telle diffusion et eut une telle influence dans le monde littéraire français. Une telle allégation ne constitue pas un cas isolé dans l'histoire de la traduction ${ }^{2}$ :

Ce sont les traductions et non les originaux qui ont valu à quelques-uns des textes les plus fameux de l'humanité leur influence exceptionnelle : les Diaboliques de Platon, la Bible, les contes d'Andersen.

Comme le précise Paul Horguelin dans son cours d'Histoire de la traduction, la réputation de Baudelaire plonge sans doute ses racines dans la critique des plus élogieuses que fit son ami Théophile Gautier de ses traductions et dans laquelle il mentionne que Baudelaire possédait à fond la langue anglaise. Toutefois, quoi qu'en dise Gautier, Baudelaire ne "possédait " pas la langue anglaise, ce qui est d'ailleurs une lacune fréquente chez les écrivains traducteurs français. Il commet des anglicismes et des calques, confond souvent l'imparfait et le passé simple et va jusqu'à supprimer des membres de phrase qu'il ne comprend pas. Julien Green, que ces déficiences agace, consigne notamment dans son Journal :

Continué la lecture de Poe dans la traduction de Baudelaire, avec une admiration qui serait sans bornes si certaines fautes ne m'arrêtaient pas. Dans l'esprit d'un Français, que peut vouloir dire une ligne d'abeille (expression des plus courantes en Amérique : a bee-line signifie une ligne parfaitement droite) ?

Il n'en reste pas moins que, en dépit de leurs fautes « techniques " (contresens, erreurs, etc.), les traductions de Baudelaire sont remarquables par leur respect de la tonalité de l'œuvre originale et leur unité de style ... le style de Baudelaire! 
À l'autre extrémité du spectre des littéraires actifs, nous avons Marguerite Yourcenar qui, pour sa part, demeure plus fidèle à l'original et fait ce que Boillot qualifie de " traduction pédagogique » : essentiellement, elle essaie non seulement de recréer l'esprit de Virginia Woolf dans The Waves, mais aussi de transcender l'œuvre pour tenter de recréer - effectivement - le style de l'auteur et, ultimement, de faire aussi bien que son modèle en faisant la même chose, en mettant en œuvre les mêmes tours, les mêmes constantes, en reproduisant les mêmes effets. Sa traduction révèle sa capacité de percevoir avec acuité les structures tant superficielles qu'occultes du texte de Virginia Woolf. Peut-on alors affirmer avec certitude que Yourcenar ait procédé à coup sûr à une analyse exhaustive du texte à traduire ? Certes pas, mais il est indubitable que sa traduction reflète fidèlement l'enveloppe stylistique de l'original, ce qui ne peut être mis sur le compte d'une simple coïncidence.

D'entrée de jeu, nous pouvons remarquer que Yourcenar s'efforce de respecter l'extraordinaire dominante de sifflantes et de liquides que l'on retrouve en anglais dans le roman de Woolf. On peut avant tout se demander si une telle dimension est pertinente : qu'il suffise de préciser que la dominante existe en anglais. On peut, si l'on veut considérer les choses de manière superficielle, n'y voir que le procédé poétique de l'allitération cher aux poètes russes et n'entendre dans "Bright arrows of sensation shoot on either side " (Woolf 1976:19) qu'une imitation onomatopoïtique du sifflement du vent de la mer, thème récurrent dans l'ouvrage. On peut aussi ne voir, dans "I tie my pajamas loosely round me, and lie a film of water drawn upon my eyes by a wave" (Woolf 1976 : 19) qu'une accumulation de liquides rappelant de l'eau qui coule... Comme l'utilisation de tels phonèmes est un reflet du fond, nous estimons que leur pertinence est indiscutable.

Dans la traduction, Yourcenar réussit assez bien à préserver la dimension phonique : " $L$ 'eau ruisselle le long de la rigole de mon épine dorsale. [...] De lourdes et puissantes sensations ruissellent sur mon cerveau comme sur un toit " (Woolf 1974: 34-35). Sur le plan stylistique, le rythme de l'original est respecté. En effet, Yourcenar s'est efforcée de préserver la longueur des phrases : "My turn has come, I come now " (Woolf $1976: 19$ ) devient : "Mon tour est venu. J'arrive. "(Woolf $1974:$ 34-35), tandis que les phrases très longues demeurent, dans la traduction très longues. La traductrice n'a pas pris sur elle de les couper " pour que le lecteur francophone puisse reprendre son souffle", argument fréquemment invoqué dans le cadre des cours de traduction.

Par souci de concision, nous nous bornerons à énoncer ces seules dimensions. En effet, il serait trop long de procéder à une critique exhaustive du texte de Yourcenar en le comparant à l'original. Il demeure que les choix lexicaux, les registres dénotatif et connotatif ont été respectés, dans la majorité des cas. Bref, à notre avis, il s'agit d'une traduction qui, si elle privilégie des dimensions différentes de celles des traductions de Baudelaire, n'en demeure pas moins excellente. Est-ce la (ou une) meilleure façon de traduire? Il ne nous appartient pas d'en juger. Les deux types ont chacun leurs mérites. En ce qui nous concerne, nous voulons faire ressortir ici que la traduction de Yourcenar n'est pas ce que nous qualifierions de traduction «instinctive ", étiquette que, par contre, l'on pourrait fort bien apposer sur celle de Baudelaire.

Passons maintenant à la catégorie constituée de ce que nous appellerons les «littéraires passifs ». Notons d'abord que nous posons en postulat que les littéraires actifs et les littéraires passifs partagent un même bagage culturel et intellectuel. Pour les fins du présent article, nous scindons cette catégorie de littéraires passifs en deux souscatégories : d'une part, les personnes n'ayant pas de formation "officielle" (par "officielle ", nous entendons non autodidacte) en traduction, et, d'autre part, ceux qui possè- 
dent une telle formation ou qui espèrent l'acquérir. Attachons-nous tout d'abord à la catégorie du traducteur n'ayant aucune formation " officielle ", dont Gregory Rabassa constitue l'exemple type. Cet excellent traducteur de romans sud-américains (Hopscotch, de Cortazar et surtout One Hundred Years of Solitude, de Gabriel Garcia Marquez) est en effet un universitaire dont la création littéraire n'est pas l'activité principale. À l'échelle internationale, la réputation de M. Rabassa n'est plus à faire et, d'ailleurs, Gabriel Garcia Marquez lui-même ne tarit pas d'éloges à son sujet. L'auteur, qui lit l'anglais, a même déclaré à Alastair Reid qu'il préférait la version traduite à l'original (Tannenbaum). C'est de loin le plus grand compliment que l'on puisse faire à un traducteur.

Malheureusement, il n'en va pas de même dans tous les cas. En effet, Milan Kundera, auteur de la Plaisanterie, souligne dans un texte intitulé "La plaisanterie était amère " qu'après la lecture de la traduction française de son roman, il était stupéfait : "le traducteur [...] n'a pas traduit le roman, il l'a écrit " (Kundera $1985: 51-52$ ). En effet, le traducteur ne s'était pas contenté d'introduire une centaine de métaphores embellissantes, de complètement dénaturer les niveaux de langue, de ne tenir aucun compte des choix lexicaux de l'auteur, préférant des formules alambiquées, il avait aussi " suivi de façon démesurée la fameuse règle du 'beau style' qui interdit la répétition du même mot. J'ai toujours exécré cette règle. En outre, la répétition donne à mon texte un rythme, une mélodie qui, dans la traduction, disparurent complètement " (Kundera 1985 : 51-52).

À la lumière de critiques de ce genre, il est plus facile de déterminer le "sens " que l'auteur souhaite voir le traducteur préserver. Ainsi, nous reviendrons sur les lacunes cernées par Milan Kundera en essayant de fournir une marche à suivre qui, dans l'opération traduisante, permettra idéalement de parvenir à une fidélité optimale tant à l'auteur qu'à son ouvre, qu'à l'esprit de son œuvre. C'est, selon nous, l'enjeu de la pédagogie de la traduction littéraire.

La deuxième sous-catégorie (parmi les littéraires passifs) regroupe les personnes qui possèdent une formation "officielle " en traduction. Rappelons qu'elles possèdent aussi, au départ, un bagage culturel et intellectuel suffisant. C'est dire qu'elles sont sensibilisées aux allusions culturelles, littéraires, religieuses, bibliques, plus particulièrement. En effet, elles doivent posséder ce que les professeurs de littérature ont toujours appelé "le fond de la langue ", savoir les dictons, proverbes, aphorismes, expressions stéréotypées, citations usuelles, etc. Comme le souligne Irène de Buisseret (1975 : 312), "Langue, culture, mentalité, tout se tient en effet, et un bon traducteur ne saurait dissocier ces éléments. "Il va sans dire que si le candidat traducteur ne possède pas ce bagage au départ, ce n'est pas dans le cadre d'un cours de traduction littéraire, ni même dans l'ensemble d'un programme de traduction que le pédagogue peut espérer le lui faire acquérir. Tout au plus peut-il lui suggérer les lectures propres à le mettre sur la bonne voie. De façon globale, donc, le rôle du pédagogue consistera à inculquer au candidat des techniques d'analyse du discours et du maniement de la langue qui permettront à ce dernier de combler son manque d'« instinct "... jusqu'à ce que l'application de ces techniques devienne chez lui un instinct.

L'objectif de la pédagogie devrait être d'aider l'étudiant à prendre conscience des ambiguïtés du discours littéraire et à perfectionner son propre style, mais sans privilégier ce dernier au détriment de l'auteur à traduire. Les aptitudes rédactionnelles varient considérablement selon les étudiants, et c'est là l'un des aspects les plus complexes à apprécier pour l'enseignant. Le critère le plus utile - et aussi le plus difficile à appliquer - est l'évaluation de l'aptitude de l'étudiant à déterminer lui-même la mesure dans laquelle il traduit fidèlement le style de l'original. S'il y parvient, en dépit de certaines ma- 


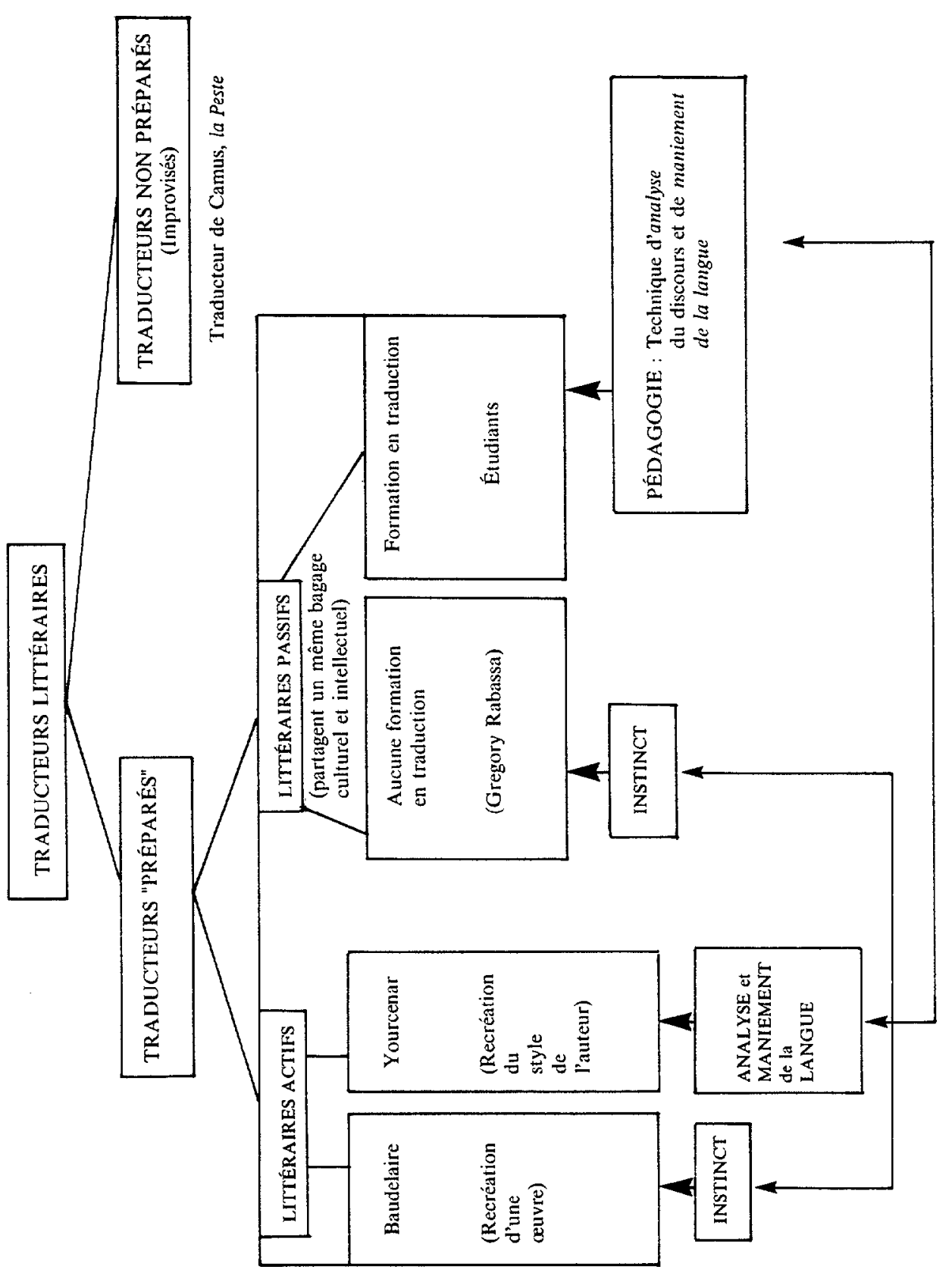


ladresses de style dues à ses propres lacunes stylistiques, alors le pédagogue doit ne tenir aucun compte de ces "faiblesses" sous réserve, bien sûr, que le texte ne soit entaché d'aucune erreur de grammaire ni de syntaxe. C'est probablement là l'un des pires obstacles qu'ait à surmonter l'enseignant de traduction littéraire ; trop souvent, il fait porter l'accent (de même que la note) sur des défaillances stylistiques en dépit du fait que, aux fins de l'exercice, savoir la traduction du texte dans un cadre d'apprentissage, le style de l'étudiant, même s'il n'est pas " prisé » par l'enseignant ou que le texte n'est pas «publiable ", convient néanmoins aux fins pédagogiques de l'exercice.

Mais à quoi tiennent ces " ambiguités du discours littéraire " ? Nous avons cerné dix dimensions auxquelles devrait s'attacher plus particulièrement l'enseignant de traduction littéraire : 1) le registre dénotatif, 2) le registre figuratif, 3) le registre connotatif, 4) le registre lexical, 5) le registre phonique, 6) les niveaux de langue, 7) les jeux de langage, 8) l'ambiguïté proprement dite, 9) la néologie et 10) les considérations biographiques.

\section{LE REGISTRE DÉNOTATIF}

Un terme est avant tout caméléon. Le terme lui-même n'a pas de couleur si ce n'est celle que lui confere le contexte. Malheureusement, les exemples d'étudiants qui voient dans le dictionnaire une panacée abondent. Ces étudiants décident que, du fait même que le terme qu'ils ont trouvé est imprimé dans un dictionnaire, cela lui confêre un caractère universel et qu'il peut se glisser indifféremment dans tous les contextes : technique, médical, juridique, cabalistique ou pornographique ! Un exemple (extrême, nous en convenons) peut être tiré du Grand Meaulnes, d'Alain Fournier (1971 : 69) :

Dans les couloirs s'organisaient des rondes et des farandoles. Une musique, quelque part, jouait un pas de menuet... Meaulnes, la tête à demi cachée dans le collet de son manteau, comme dans une fraise, se sentait un autre personnage.

Les paradigmes contextuels dans l'extrait précité, voire dans l'ensemble du chapitre, sont d'abord celui des vêtements, et ensuite celui des costumes. Or, plutôt que de $s$ 'inspirer de ces paramètres, quelques excellents étudiants, qui en étaient à la dernière année d'un programme de traduction échelonné sur quatre ans, ont mis leur foi en l'omnipotence du dictionnaire et, partant, ont fourni les traductions suivantes :

a) ...his head like a strawberry-in-a-poke...

b) ...his head, strawberry-like, emerged from his cloak...

c) ...his head, counter-sunk in his cloak...

Le caractère grotesque des traductions n'est éclipsé que par l'ingénuité dont ont fait preuve les étudiants en déformant le contexte pour l'adapter à grand-force à la solution unidimensionnelle qu'ils avaient puisée dans le dictionnaire. En effet, le terme "fraise " désigne, outre le fruit (strawberry), une collerette (ruff) et tout un assortiment d'outils de forme conique ou cylindrique (d'où counter-sunk). Les trois traductions surréalistes qui ont été fournies sont d'autant plus surprenantes lorsqu'on se rend compte que le roman n'a rien de surréaliste (tout au plus renferme-t-il quelques passages où le lecteur sombre dans l'imaginaire) ; l'image soudaine et apparemment gratuite de la tête de Meaulnes transformée en une fraise n'a aucunement troublé les étudiants : la loi du dictionnaire est telle qu'elle peut donner lieu à une gymnastique linguistique des plus extraordinaires sans même inciter l'intéressé à mettre un frein à son imagination et à sonder le contexte. Pourquoi le ferait-il ? Le mot a été "trouvé dans le dictionnaire" !

Il convient de souligner que les étudiants en question étaient loin d'être des débutants : tous étaient expérimentés, certains étaient des professionnels. Seulement, pour quelque mystérieuse raison, la raison ne les habitait plus. Que s'était-il produit ? Ils 
avaient perdu cette sensibilisation contextuelle, cette conscience du paradigme contextuel, sans laquelle le traducteur d'un texte littéraire travaille à l'aveuglette, un peu comme un pilote qui vole sans visibilité.

\section{LE REGISTRE FIGURATIF}

La dimension figurative n'est pas la même chose que la dimension connotative : nous entendons par figuratif le registre de la langue dans lequel un terme a perdu son acception concrète et ne peut être employé, contrairement au terme correspondant dans la langue source, qu'au figuré. Soulignons en outre que nous ne faisons pas rentrer dans cette catégorie les termes ayant une acception tant concrète qu'abstraite (par exemple, tuer quelqu'un et tuer le temps / to kill time et to kill somebody). Voici un exemple tiré de Noces à Tipasa, de Camus (1962:14) :

Au bout de quelques pas, les absinthes nous prennent à la gorge. Leur laine grise couvre les ruines à perte de vue. Leur essence fermente sous la chaleur, et de la terre au soleil monte sur toute l'étendue du monde un alcool généreux qui fait vaciller le ciel.

Le verbe " vaciller » ne peut être rendu par to vacillate, qui a perdu toute acception concrète et, par conséquent, l'effet de sens devra être véhiculé d'une autre façon (par exemple, par le verbe to shimmer dans le cas qui nous occupe).

\section{LE REGISTRE CONNOTATIF}

Il est primordial de tenir compte de la dimension connotative lorsqu'il s'agit de comprendre et de traduire un texte littéraire. À cet égard, l'efficacité de la pédagogie sera manifestement tributaire de l'étendue du bagage culturel et intellectuel de l'étudiant. Ainsi, le rôle du pédagogue se bornera à stimuler la sensibilisation de l'étudiant face au texte et à souligner le(s) paradigme(s) contextuel(s). S'il est relativement simple de sensibiliser l'étudiant à la dimension lexicale, ce dernier ne pourra prendre conscience d'une dimension connotative qui s'articule sur des tours syntaxiques précis qu'après avoir effectué les recherches voulues. Le candidat traducteur doit se demander, par exemple, pourquoi Camus (1962:16) a choisi de tourner ainsi une phrase au début d'un paragraphe truffé d'allusions à des pratiques religieuses païennes :

Bien pauvres sont ceux qui ont besoin de mythes. Ici les dieux servent de lits ou de repères dans la course des journées.

Camus joue ici sur deux registres : païen et chrétien. Partant il est essentiel que le traducteur fasse en sorte que le tour syntaxique qu'il utilisera soit modelé sur la formulation des Béatitudes (tournure qui est facile à reproduire en anglais).

Quelquefois, les allusions connotatives sont à ce point subtiles qu'elles peuvent échapper même à l'œil de lynx d'une Yourcenar. Dans The Waves, de Virginia Woolf (1976: 161), Bernard utilise, en parlant de sa vie, un tour syntaxique tiré directement de la Bible et du missel :

This, for the moment, seems to be my life. If it were possible, I would hand it to you entire. I would break it off as one breaks off a bunch of grapes. I would say 'Take it. This is my life.'

Yourcenar (1974:257) rend le passage en question (c'est nous qui soulignons) par :

Prenez. C'est cela ma vie.

plutôt que d'utiliser " Ceci est ma vie ", ce qu'elle aurait dû faire si elle avait voulu reproduire l'évocation liée à la syntaxe du texte anglais : en effet, on aurait reconnu la parole 
que prononce le prêtre au moment de la consécration, la même que le Christ avait prononcée au cours de la Cène.

\section{LE REGISTRE LEXICAL}

Dans les textes littéraires, les choix lexicaux sont tout aussi importants que les choix terminologiques que doit faire le candidat traducteur qui s'attaque à un texte technique. Toutefois, dans le domaine littéraire, l'accent doit porter sur le lexique personnel de l'auteur, sur son paradigme idiosyncrasique. L'étudiant doit faire des recherches approfondies à cet égard. Il n'est pas nécessaire de parcourir toute l'œuvre de l'auteur, mais il faut lire attentivement plusieurs passages représentatifs. Or, la pratique de la traduction devient un moyen privilégié d'être sensible à la praxis verbale de l'auteur à traduire. Il va de soi que chaque écrivain et, à plus forte raison, chaque poète, se meut dans son champ lexical bien déterminé où les mots pris isolément n'ont rien de particulier. Toutefois, il est intéressant de constater avec Ivan Fonagy, Jean-Pierre Richard (1961) et d'autres que les éléments qui définissent un champ lexical, pris dans leur ensemble, constituent une aire métaphorique propre à chaque écrivain. C'est ce champ sémique spécifique qu'il faudrait explorer en vue de dégager les mots clefs que l'on retrouve enchâssés dans l'œuvre à traduire. Dans cet espace privilégié qu'est la traduction, il s'agit de trouver le point stratégique d'où l'on peut apercevoir le paysage métaphorique spécifique; ce point où la lecture de l'œuvre en langue étrangère - quelle qu'elle soit - produirait la même réponse affective (Iser 1974) que celle éprouvée par le lecteur de l'œuvre originale.

Il est donc essentiel pour le traducteur de compiler un lexique des mots clefs, de les traduire et d'y adhérer fermement dans sa traduction sans recourir à la panoplie habituelle de synonymes, d'équivalents, de variantes et de périphrases à laquelle tout traducteur, dans d'autres circonstances, serait tenté de recourir (Slote 1982). Une fois dégagée, cette configuration sémique doit conserver sa dimension "obsessionnelle", de façon à éviter la déficience même que déplorait Milan Kundera (1985) : «...la répétition donne à mon texte un rythme, une mélodie qui, dans la traduction, disparurent complètement. »

\section{LE REGISTRE PHONIQUE}

La dimension phonique est sans conteste l'une de celles dont on fait le plus souvent abstraction. En effet, trop souvent, le candidat traducteur pose en hypothèse qu'elle ne joue un rôle que dans le cas d'une œuvre poétique. En réalité, nombre d'écrivains l'exploitent dans des œuvres rentrant dans toute la gamme des genres littéraires. Ainsi, le traducteur sera bien inspiré d'appréhender le texte de cet angle : constate-t-il une occurrence marquée de phonèmes d'un même type (occlusives, nasales, sifflantes, par exemple), ou encore, ces phonèmes brillent-ils par leur absence? Si tel est le cas, l'étudiant ne peut guère que le remarquer. Toutefois, à partir du moment où il a isolé une telle dominance ou absence, il doit en établir la pertinence avant de la récupérer pour véhiculer le même effet de sens dans sa traduction. Pour ce faire, il doit établir un lien assez net entre le fond et la forme. En effet, il doit se demander si les phonèmes dominants évoquent un thème principal ou secondaire de l'œuvre, si une absence de phonèmes d'un type donné est " logique » du fait que ces phonèmes sont inconciliables avec le thème dominant, qu'ils désamorceraient. Ainsi, chez Woolf, comme nous l'avons précisé plus haut, les liquides, fort abondantes, étaient étroitement liées au thème central de l'œuvre, l'eau, tandis que les sifflantes évoquaient le thème secondaire, le vent de la mer.

Marguerite Yourcenar a-t-elle vu dans cet effet de style la seule manifestation de l'allitération ou a-t-elle poussé plus loin et entrepris une véritable analyse de l'œuvre de Woolf axée sur cette dimension? Nous ne saurions dire, mais en tout état de cause, il est 
indiscutable qu'elle a préservé la dominance de sifflantes et de liquides dans maints passages de la traduction. À coup sûr, comme le font ressortir les travaux de Fonagy (1970) et de Peraldi $(1984,1985)$, une telle accumulation de phonèmes a sur le lecteur un effet de sens marqué, effet qui transcende les barrières linguistiques.

Mais l'auteur introduit-il consciemment cette dimension dans son cuvre? Il ne nous appartient pas de le déterminer : il demeure que, consciemment ou non, il l'y a mise ; c'est l'essentiel. Certains auteurs, du reste, sont explicites : dans Molloy, Beckett explique ainsi le choix du nom qu'il donne à sa mère (Peraldi 1984) :

Moi je l'appelais Mag quand je devais lui donner un nom. Et si je l'appelais Mag, c'était qu'à mon idée, sans que j'eusse su dire pourquoi, la lettre g abolissait la syllabe ma, et, pour ainsi dire, crachait dessus mieux que toute autre lettre ne l'aurait fait...

\section{LES NIVEAUX DE LANGUE}

L'un des problèmes majeurs auxquels est confronté le traducteur est le respect de la tonalité du texte, des variations de niveaux de langue. Ainsi, le candidat traducteur doit maîtriser les divers niveaux de langue : littéraire (relevé), tenu (ou d'exposition), familier, populaire et argotique, en dépit du fait que la démarcation entre deux niveaux voisins ne soit pas toujours nette, de crainte de déniveler et de commettre certains impairs. D'après Mr. Ronald Knox, dans On English Translation (de Buisseret 1975 : 137) :

A good translation does not demand a good reproduction of detail, but it does demand a certain identity of atmosphere.

Les écarts de niveau peuvent provoquer des réactions diverses. Le destinataire du message peut avoir une réaction négative, "soit qu'il interprète l'emploi d'un niveau trop relevé comme l'indice d'une attitude distante ou méprisante, soit, au contraire, qu'il considère comme déplacé ou démagogique le recours à un niveau familier ou populaire " (Horguelin 1985 : 162). Toutefois, l'écrivain qui joue sur les niveaux de langue campe ainsi ses personnages. Il importe évidemment pour le candidat traducteur de respecter le niveau propre à chaque personnage de façon à ne pas dénaturer le caractère de chacun. Néanmoins, il arrive que certaines dénivellations soient voulues par l'auteur et créent un effet comique. Par exemple, dans le célèbre roman de J.D. Salinger, The Catcher in the Rye, Holden Caulfield s'exprime habituellement dans une langue bien à lui, qui oscille entre les niveaux familier, populaire et argotique. Cependant, lorsqu'il dialogue avec un de ses anciens professeurs (il vient d'être renvoyé), il essaie de se mettre au diapason de ce dernier et n'y réussit qu'imparfaitement :

- Has Dr. Thurmer written to your parents yet?

- He said he was going to write them Monday.

- Have you yourself communicated with them?

- No, sir, I haven't communicated with them, because I'll probably see them Wednesday night when I get home.

- And how do you think they'll take the news?

- Well... they'll be pretty irritated about it.

On constate dès le départ l'écart qui sépare le niveau de langue du professeur Spencer de celui de Holden Caulfield. Par contre, Holden s'efforce de « bien parler » («I haven't communicated with them [...] they'll be pretty irritated "). Mais comme il ne mấtrise pas ce niveau de langue, la dénivellation est très nette. Dans l'expression " pretty irritated », les deux termes accolés appartiennent à des niveaux diamétralement opposés : en effet, Holden aurait dit spontanément " they'll be pretty mad, pretty sore, pretty pissed 
off ", ou quelque autre expression du même niveau et aurait évité comme la peste "irritated", qui est beaucoup trop relevé.

Pour le traducteur, un autre problème se pose : l'absence d'un niveau familier ou populaire en français dit "international ", c'est-à-dire un niveau désincarné, " passepartout ", qui ne soit pas fortement marqué par des éléments propres à un contexte à travers le monde, l'argot est le propre de la France, et le joual, l'apanage du Québec. Toutefois, à des fins purement pédagogiques, l'enseignant pourra choisir de préciser d'entrée de jeu le public auquel s'adresse la traduction. Il va sans dire que cette façon de faire n'est valable qu'en milieu d'apprentissage et à des fins pédagogiques : d'aucuns allégueront que l'on ne saurait transplanter décemment Holden Caulfield en milieu québécois ou le faire s'exprimer en titi parigot sans que la substance même de l'ouvre n'en souffre. Une telle traduction, si elle constitue un exercice des plus profitables, ne serait pas forcément publiable.

\section{LES JEUX DE LANGAGE}

Combien de fois a-t-on entendu les gens se gargariser de l'allégation suivante : "Les jeux de mots, ça ne se traduit pas! "? Une telle affirmation, si elle était fondée, remettrait en question, notamment, tous les efforts déployés par les traducteurs des œuvres de Lewis Carroll, principalement Alice in Wonderland et Through the LookingGlass. Il existe effectivement en français une quarantaine de versions différentes, aussi bien traductions qu'adaptations d'Alice. Si les traductions sont plus ou moins heureuses, il demeure que nombre des problèmes présents dans l'original - nous nous préoccupons ici au premier chef des jeux de langage - ont été souvent réglés de façon satisfaisante. Abstraction faite des problèmes culturels liés à la traduction, qu'a étudiés Claude Romney (1984:267-280), une forte proportion de jeux de mots apparemment intraduisibles ont été rendus, à notre avis, de façon pleinement satisfaisante. Alice in Wonderland renferme un certain nombre de proverbes déformés par l'auteur dans un but humoristique et dont la traduction s'avère malaisée. Ainsi, lorsque le Roi de Cœur réprimande le Chat du Cheshire qui a refusé de lui baiser la main, il lui lance :

Don't be impertinent [...] and don't look at me like that!

Alice prend la défense du Chat :

$A$ cat may look at a king, [...] I've read that somewhere, but I don't remember where.

Le problème, évidemment, est le suivant : en français, l'équivalent serait : "Un chien regarde bien un évêque. » Si le traducteur se contente de rendre littéralement le proverbe anglais, le lecteur français ne reconnaît pas l'expression proverbiale. Il va sans dire que le traducteur ne peut mettre dans la bouche d'Alice le proverbe français, dont les deux personnages n'ont rien à voir avec le contexte. Henri Parisot, pour sa part, accole avec adresse les deux dictons (Romney 1984) :

Pourquoi, dit Alice, un chat ne regarderait-il pas un roi ? Un chien regarde bien un évêque !

Lorsqu'il est confronté à un problème de ce genre, le candidat traducteur doit d'abord déterminer de quelle façon s'articule le jeu de langage. Ainsi, dans Through the Looking-Glass, lorsque Alice se retrouve dans je jardin des fleurs vivantes, elle converse en ces termes avec les fleurs :

"Aren't you sometimes frightened at being planted out here, with nobody to take care of you?"

"There's the tree in the middle, " said the Rose. "What else is it good for?" 
"But what could it do if any danger came?" Alice asked.

"It could bark, " said the Rose.

"It says 'Bough-wough!' " cried a Daisy : "that's why its branches are called boughs!"

Le problème, ici, consiste à reproduire, dans un premier temps, le lien entre tree et bark (écorce/aboyer) et, dans un deuxième temps, à pouvoir greffer (si l'on peut dire) le second jeu de mots : bough-wough (onomatopée correspondant à un aboiement) et boughs (rameaux de l'arbre, branches). L'étudiant remarquera sans mal que tous ces termes proviennent du même paradigme - celui de l'arbre et de ses composantes. Pour recréer le jeu de mots, il peut chercher à établir une liste de termes rentrant dans ce paradigme et pouvant avoir une deuxième acception qui, elle, se retrouverait dans le deuxième paradigme : le danger, la protection. S'il ne trouve pas de termes sur lesquels il puisse articuler un jeu de langage équivalent, il agrandit l'aire de son premier paradigme, par exemple en y faisant rentrer les noms d'arbres. Certaines possibilités s'offrent :

saule - pleurer (saule pleureur)

marronnier - donner des marrons (pop. coup de poing)

chêne - dé-chêner.

C'est pour cette dernière possibilité que Henri Parisot a opté dans sa traduction. La première ne saurait convenir puisque pleurer ne fait pas partie, lui, du second paradigme : en effet, le fait de pleurer n'a jamais protégé qui que ce soit (et cela est valable même au Pays des Merveilles). La deuxième possibilité n'est pas mauvaise, mais elle présente comme inconvénient un changement de niveau de langue.

Il reste à régler le deuxième jeu de langage. Parisot s'en sort de la façon suivante, en expliquant la graphie peut-être un peu osée :

Cela lui arrive parfois, confirme une Pâquerette. Si on le met hors de lui, il cesse sur-lechamp d'être un chêne et l'on peut donc dire qu'il se dé-chêne!

Des étudiants à qui l'on avait donné le même texte à traduire ont pensé à poursuivre dans le même paradigme que l'anglais et ont proposé que l'arbre/le chêne mette les intrus à l'ombre. Abstraction faite du léger glissement de niveau de langue, cette solution s'insère très bien dans le texte et s'inspire directement du procédé utilisé en anglais. Le pédagogue devra donc sensibiliser, une fois de plus, l'étudiant aux ressources de l'axe paradigmatique. Une fois qu'il a isolé les paradigmes en présence, le candidat traducteur n'a plus qu'à chercher les termes ou syntagmes qui chevauchent ces paradigmes.

\section{L'AMBIGUÏTÉ}

Dans ce registre, le pédagogue doit insister sur une considération qu'il sera difficile pour l'étudiant de saisir et d'appliquer, particulièrement pour les candidats traducteurs dont la formation ou les antécédents professionnels sont concentrés en traduction technique. Comme l'énonce Rimbaud dans sa Lettre dite du Voyant :

Si ce qu'il [le poète] rapporte de là-bas a forme, il donne forme ; si c'est informe, il donne de l'informe. Trouver une langue !

Cet énoncé peut s'appliquer à la traduction dans la mesure où les contextes équivoques ou ambigus (en raison des tours syntaxiques ou des éléments lexicaux utilisés) doivent être laissés « informes", c'est-à-dire que l'ambiguïté doit être reproduite dans la 
traduction. Ce n'est pas là une tâche facile. Prenons, par exemple, le passage suivant, où Colette (1973) décrit sa mère :

Je l'ai vue suspendre, dans un cerisier, un épouvantail à effrayer les merles [...] Peu de jours après, je trouvais ma mère sous l'arbre, passionnément immobile, la tête à la rencontre du ciel d'où elle bannissait les religions humaines...

- Chut !... Regarde...

Un merle noir, oxydé de vert et de violet, piquait les cerises, buvait le jus, déchiquetait la chair rosée...

- Qu'il est beau !... chuchotait ma mère. Et tu vois comme il se sert de sa patte ? Et tu vois les mouvements de sa tête et cette arrogance ? Et ce tour de bec pour vider le noyau ? Et remarque bien qu'il n'attrape que les plus mûres...

- Mais, maman, l'épouvantail...

- Chut !... L'épouvantail ne le gêne pas...

- Mais, maman, les cerises!...

Ma mère ramena sur la terre ses yeux couleur de pluie :

- Les cerises ?... Ah ! oui, les cerises...

Dans ses yeux passa une sorte de frénésie riante, un universel mépris, un dédain dansant qui me foulait avec tout le reste, allégrement... Ce ne fut qu'un moment unique. Maintenant que je la connais mieux, j'interprète ces éclairs de son visage. Il me semble qu'un besoin d'échapper à tout et à tous, un bond vers le haut, vers une loi écrite par elle seule, pour elle seule, les allumait. Si je me trompe, laissez-moi errer.

Sous le cerisier, elle retomba une fois parmi nous, lestée de soucis, d'amour, d'enfants et de mari suspendus, elle redevint bonne, ronde, humble devant l'ordinaire de sa vie :

- C'est vrai, les cerises... Il te faut aussi les cerises...

Outre les problèmes lexicaux (frénésie riante... dédain dansant... d'enfants et de nari suspendus... ronde...), on remarque une caractéristique intéressante et subtile de son texte : en jouant sur les temps de verbe, Colette essaie de communiquer au lecteur le sentiment qu'il s'agit là d'une anecdote unique, isolée (lorsqu'elle utilise le passé simple) et, dans un même temps, de laisser supposer que cette scène s'est probablement répétée chaque été (lorsqu'elle utilise le passé composé et l'imparfait). Le candidat traducteur doit être sensibilisé à ce jeu sur les temps de verbe s'il veut conserver l'ambiguité temporelle qu'a créée Colette. Cette ambiguïté fait partie intégrante du message ; l'étudiant doit la préserver intégralement, sans chercher à " préciser les choses " à l'intention du lecteur de sa traduction.

Par contre, l'ambiguité est le plus souvent d'ordre lexical. Ainsi, dans Surfacing, Margaret Atwood (1972:160) fait décrire à la narratrice un champignon en ces termes:

... further along a mushroom with cup and ring and chalk gills and a name: Death Anger, deadly poison. Beneath it, the invisible part, threadlike underground network of which this was the solid flower, temporary as an icicle, growth frozen; tomorrow it would be melted but the roots would stay.

Comme, dans tout le paragraphe en question, la narratrice évoque la vie, la mort, l'évolution, un problème se pose : growth renvoie-t-il à la croissance ou à une excroissance ? Dans le texte de départ, l'image est d'autant plus riche, plus puissante, que cette ambiguïté joue. C'est pourquoi le traducteur sera bien inspiré de ne pas trancher et d'opter pour un terme comme "pousse", qui a les deux acceptions : " action de pousser et ce qui pousse à un certain stade de la végétation; specialt. Bourgeon naissant, jet [...]" (Dictionnaire Robert : v. pousse). Malheureusement, il est des cas où même la recherche la plus poussée ne permettra pas au traducteur de préserver l'ambiguïté : il doit alors trancher, mais en s'appuyant sur d'autres données : interprétation globale de l'œuvre, 
considérations biographiques, etc. C'est ce qui se produit dans la traduction de Dévotion, de Rimbaud. Rimbaud (1967) termine son poème en ces mots :

Mais plus alors.

Comme le précise le traducteur (Slote 1978 : 95), Rimbaud ayant souligné "alors", il s'agit probablement d'une indication relative au niveau de langue (comme dans l'expression : Ah ça, alors!) Problème suivant : « alors " est-il temporel ou séquentiel ? La polysémie du terme est telle que le poème se termine sur une note de parfaite ambiguité. Le nombre d'interprétations est presque illimité. Le traducteur s'efforce de respecter un maximum de niveaux, mais il est bien conscient qu'il y aura inéluctablement perte. Autre problème : "plus». Nous avons ici, encore une fois, une ambiguïté parfaite : si le " $s$ » final est prononcé, le terme prend un sens diamétralement opposé au sens qu'il a si ce " $s$ " n'est pas prononcé. Voilà, selon nous, un exemple des plus évocateurs d'une ambiguïté insoluble.

\section{LA NÉOLOGIE}

En littérature, les exemples de création néologique foisonnent. L'un des genres littéraires les plus susceptibles de faire appel à ce procédé est sans conteste la sciencefiction, l'écriture fantastique. Un auteur contemporain qui en fait largement usage est Kurt Vonnegut, Jr. L'exemple selon nous le plus percutant nous est fourni par le titre d'un de ses ouvrages : Wampeters, Foma and Granfalloons, qu'il décrit en ces termes dans la préface $(1976: x v)$ :

Dear Reader :

The title of this book is composed of three words from my novel Cat's Cradle. $A$ wampeter is an object around which the lives of many otherwise unrelated people may revolve. The Holy Grail would be a case in point. Foma are harmless untruths, intended to comfort simple souls. An example: "Prosperity is just around the corner." A granfalloon is a proud and meaningless association of human beings.

Face à trois néologismes de ce genre, comment doit réagir le candidat traducteur? Son premier réflexe doit évidemment consister à établir si le choix du néologisme est motivé. Comme chaque néologisme est accompagné d'une définition, il peut dès l'abord le soupçonner. Dès lors, il ne lui reste qu'à scinder les divers termes de façon à déterminer de quels morphèmes ils sont constitués. Ainsi, dans le cas de wampeter, il pourra peut-être isoler wamp- (de wampum : monnaie indienne) et -eter (de eater, qui évoquerait l'appât du gain). En ce qui a trait à foma, les choses se compliquent. Il peut isoler :

$f$-de alse (untruths)

fo-de phoney (untruths)

fom-de foam (mousse, écume, qui a peu de substance) (harmless)

$-a$ suffixe latin pluriel

-ma de dogma (untruths - to comfort simple minds)

-oma de soma ${ }^{3}$ (comfort)

C'est plus qu'il ne lui en faut; de toute façon, chacune des possibilités trouve un

reflet dans la définition fournie par l'auteur. Passons maintenant à grandfalloons :

gran- de grandiose (proud)

fal- de fallacy (meaningless)

-loon de loonies (meaningless human beings)

ou : alloon de balloon (idem à ci-dessus plus évocation de hot air)

Une fois qu'il a procédé à une telle analyse des morphèmes constitutifs, l'étudiant n'a plus qu'à trouver d'autres radicaux et d'autres terminaisons qui, dans la langue ci- 
ble, sauront véhiculer avec autant d'évidence le «sens » que portent les morphèmes dans la langue source.

\section{LES CONSIDÉRATIONS BIOGRAPHIOUES}

Comme l'a fait remarquer Nabokov (1955), les traductions d'auteurs russes vers l'anglais, le français ou l'allemand sont souvent mauvaises parce que le traducteur connaît mal la langue et les coutumes, l'auteur et ses sources d'inspiration. L'écrivain russe soulignait là un problème de taille : trop de traducteurs, en effet, font des recherches parfois trop superficielles sur l'auteur à traduire. D'autres encore s'attachent aux mauvais éléments. Bien des étudiants qui assistent à un exposé sur la vie et l'œuvre d'un auteur (qu'ils auront ensuite à traduire) se bornent à consigner les « dates importantes » de sa vie, le nom des amis, du (ou des) conjoint(s), amant(s), maîtresse(s), etc., sans se préoccuper de chercher un lien solide entre l'œuvre à traduire et la vie ou les opinions de l'auteur. Ainsi, le candidat traducteur qui doit s'attaquer à un texte de Colette ou de Margaret Atwood sans garder présent à l'esprit que la première était passionnée de botanique et que la seconde était très sensibilisée à cette science, ayant passé la majeure partie de son enfance et de son adolescence avec son père (entomologiste) dans le nord du Québec, risque de confondre certaines " appellations contrôlées " de plantes avec des figures de rhétorique. Ainsi, dans son roman Surfacing, Margaret Atwood (1955: 160) décrit en ces termes la végétation qui l'entoure (par l'entremise de la narratrice) :

Sight flowing ahead of me over the ground, eyes filtering the shapes, the names of things fading but their forms and uses remaining, the animals learned what to eat without nouns. Six leaves, three leaves, the root of this is crisp. White stems curved like question marks, fishcoloured in the dim light, corpse plants, inedible. Finger-shaped yellow fungi, unclassified $[\ldots]$

Si le candidat traducteur n'est pas à l'affût de ce genre de chausse-trappe - et c'est précisément l'objectif que devrait se fixer le pédagogue : de l'y sensibiliser — il sera porté à croire que l'auteur vient de créer une métaphore originale et ne fera pas les recherches qui lui permettraient de se rendre compte du fait que l'expression corpse plant renvoie à un monotrope, ainsi désigné à cause de l'absence de chlorophylle. Pour pallier cette lacune, la pédagogie devra faire porter l'accent sur l'importance de procéder à une analyse approfondie de la biographie de l'auteur, de son œuvre, des critiques et exégèses relatives à cette dernière, des influences qu'a subies l'auteur et de celle qu'il a eue sur l'ensemble de la littérature, le cas échéant.

Si nous avons traité de cette dimension en tout dernier lieu, ce n'est certes pas parce qu'elle revêt une importance moindre que les autres registres, mais plutôt du fait qu'elle est, dans une certaine mesure, paratextuelle. En réalité, il conviendrait de s'attacher d'abord et avant tout à cette recherche pour jeter les fondements de l'analyse.

\section{CONCLUSION}

À mesure que les représentants des catégories de traducteurs considérées dans le présent article influent sur le monde littéraire, que les critiques se penchent sur les traductions en ne cherchant pas une rédaction parallèle inspirée d'un original (" une traduction est bonne quand elle se lit comme l'original "), des exemples excessifs quoique délicieux comme la traduction de la Peste, de Camus, disparaissent pour faire place à des traductions plus " fouillées », qui prennent en compte un certain nombre de paramètres, notamment ceux que nous avons décrits plus haut. Notons au passage que la liste ne prétend aucunement être exhaustive, il s'en faut de beaucoup.

Idéalement, la pédagogie de la traduction littéraire devrait donc viser à stimuler une sensibilisation contextuelle et paradigmatique chez le candidat traducteur, à lui in- 
culquer quelques paramètres d'analyse : lexical, syntaxique, stylistique et phonique, qui lui permettront d'appréhender en connaissance de cause les particularités inhérentes à la praxis rédactionnelle de l'auteur à traduire. Ce n'est qu'après les lectures nécessaires (biographies, critiques, exégèses), qui constituent la pierre angulaire de toute bonne analyse approfondie, que le traducteur pourra appliquer sa grille d'analyse de façon à faire la lumière sur toutes allusions, connotations et ambiguïtés recensées dans l'œuvre et enfin s'attaquer de façon éclairée à la traduction proprement dite. En un mot, cette recherche et cette analyse forment la trame et la chaîne de la toile traductionnelle que tissera le traducteur littéraire.

Notes

1. Dans le présent article, nous utiliserons le terme "traduction littéraire" en posant en postulat que le candidat traducteur travaille toujours vers sa langue maternelle.

2. Bertil Malmberg (1978) : Introduction au Colloque, Theory and Practice of Translation, Nobel Symposium 39, Berne, Peter Lang, p. 15 (cité dans META, 23:3, page couverture).

3. Dans Brave New World, de Huxley, ce terme désigne une drogue euphorisante.

\section{BIBLIOGRAPHIE}

ATWOOD, Margaret (1972) : Surfacing, Toronto, Toronto General Publishing Co. Ltd.

BLOOMFIELD, L. (1933) : Language, New York, Holt.

CAMUS, Albert (1948) : la Peste (traduction : Gilbert Stewart), Penguin.

CAMUS, Albert (1962) : "Noces à Tipasa ", in Noces, Paris, Gallimard.

CARROLL, Lewis (1971) : Through the Looking-Glass/De l'autre côté du miroir, Paris, Aubier-Flammarion (traduction de Henri Parisot).

COLETTE (1973) : Sido, Paris, Editions G.P.

DE BUISSERET, Irène (1975) : Deux langues, six idiomes, Ottawa, Carlton Green.

DE SAUSSURE, Ferdinand (1982) : Cours de linguistique générale, Paris, Payot.

FEDOROV, A.V. (1953) : Introduction à la théorie de la traduction (Vvedenie v teoriju perevoda), Moscou, Institut des littératures en langues étrangères.

FONAGY, Ivan (1970 et 1971) : "Les bases pulsionnelles de la phonation ", Revue française de psychanalyse, vol. I et IV.

FOURNIER, Alain (1971) : le Grand Meaulnes, Paris, Fayard.

HORGUELIN, Paul A. (1981) : Anthologie de la manière de traduire, Montréal, Linguatech.

HORGUELIN, Paul A. (1985) : Structure et style, Montréal, Linguatech.

ISER, Wolfgang (1974) : The Implied Reader : Patterns of Communication in Prose Fiction from Bunyan to Beckett, Baltimore.

KUNDERA, Milan (1985) : "La plaisanterie était amère ", in le Nouvel Observateur, 1025, 23-29 août.

MOUNIN, Georges (1955) : les Belles Infidèles, Paris, Cahiers du Sud.

MOUNIN, Georges (1963) : les Problèmes théoriques de la traduction, Paris, Gallimard.

NABOKOV, Vladimir (1955): "Problems of Translation : 'Origin' in English ", in Partisan Review, no 22.

PERALDI, François (1984): "Conditions théoriques de la traduction ", in la Traduction: l'universitaire et le praticien, publié sous la direction d'Arlette Thomas et Jacques Flamand, Éditions de l'Université d'Ottawa.

PERALDI, François (1984) : "The Erotic Body of Language", communication présentée à l'occasion du Fifth International Summer Institute for Semiotics and Structural Studies, Colloquium on Semiotics of Literary Translation, Victoria University, Toronto, 23 juin.

RICHARD, Jean-Pierre (1961) : l'Univers imaginaire de Mallarmé, Paris.

RIMBAUD, Arthur (1954) : Lettre dite du Voyant, 15 mai 1871, Paris, Messein.

RIMBAUD, Arthur (1967) : Illuminations, in CEuvres complètes, Paris, Gallimard (Pléiade).

ROMNEY, Claude (1984): "Problèmes culturels de la traduction d'Alice in Wonderland en français", in META, 29:3, pp. 267-280.

SALINGER, J.D. (1964) : The Catcher in the Rye, New York, Bantam.

SLOTE, Daniel (1978) : "Translating Rimbaud's 'Illuminations' : Games with Words", in META, 23:1.

SLOTE, Daniel (1982) : "Comment traduire Rimbaud : Problèmes psycholinguistiques", in META, 27:1, pp. 129-136.

STEINER, George (1978) : Après Babel. Une poétique du dire et de la traduction, Paris, Albin Michel.

TANNENBAUM, Jeffrey A. : "The Translator's Role is Crucial and Delicate, and Widely Unnoticed ", in The Wall Street Journal, vol. CXC, $\mathrm{n}^{\circ} 53$.

VONNEGUT, Kurt (1976) : Wampeters, Foma and Granfalloons (Opinions), New York Dell. 
WOOLF, Virginia (1976) : The Waves, Toronto, University of Toronto Press.

WOOLF, Virginia (1974) : les Vagues, Paris, Stock (traduction de Marguerite Yourcenar). 